\title{
AREA-MINIMIZING OF THE CONE OVER SYMMETRIC $R$-SPACES
}

\author{
By \\ Daigo Hirohashi, Takahiro KanNo and Hiroyuki TASAKI
}

\section{Introduction}

Let $B$ denote a submanifold of the unit sphere in $\boldsymbol{R}^{n}$ and $C_{B}$ the cone over $B$, which is the union of rays starting from the origin and passing through $B$.

A cone is called area-minimizing if the truncated cone $C_{B}^{1}$ inside the unit ball is area-minimizing among all surfaces with boundary $B$. The surfaces we will use are integral currents. A tangent cone to surface $S$ at a point $p \in S$ can be thought of as the union of rays starting from $p$ and tangent to $S$ at $p$. This is the generalization of the notion of tangent plane. If the tangent cone at $p$ is not a plane, then $p$ is a singular point of $S$. If $S$ is area-minimizing, then each tangent cone to $S$ is area-minimizing. Thus in order to study area-minimizing surface with singularities, we need to know which cones are area-minimizing.

G. R. Lawlor proposed a criterion for area-minimization in [5]. His principal idea is to construct an area-nonincreasing retraction $\Pi: R^{n} \rightarrow C$. If $S$ is another surface which has the same boundary as $C_{B}^{1}$, it will follow that

$$
\operatorname{vol}(S) \geq \operatorname{vol}(\Pi(S)) \geq \operatorname{vol}\left(C_{B}^{1}\right)
$$

since $\Pi(S)$ must cover all of $C_{B}^{1}$. Using this method, he gave a complete classification of area-minimizing cones $C$ over products of spheres and the first example of minimizing cone over a nonorientable manifold. In order to construct the retraction he solved a differential equation with numerical analysis.

In this paper, we consider the canonical imbeddings of symmetric $R$-spaces which are linear isotropy orbits of symmetric pairs. Using root systems, we construct area-nonincreasing retractions concretely.

In section 1 we prepare some notation and terminology, and prove an essential theorem (Theorem 1.6) for construction of the retractions. In section 2 we describe the canonical imbeddings of symmetric $R$-spaces, and construct 
retractions onto the cones over them. In section 3 we apply the result of section 2 to symmetric $R$-spaces associated with symmetric pairs of type $B_{l}$.

Concerning the cones over symmetric $R$-spaces, B. N. Cheng [1] proved the cone over $U(n) / O(n)$ and $U(n)$ are area-minimizing in $\boldsymbol{R}^{n^{2}+n}$ for $n \geq 7$ and $\boldsymbol{R}^{2 n^{2}}$, respectively, by calibration. G. R. Lawlor [5] proved the cone over $S O(n)$ are area-minimizing in $\boldsymbol{R}^{n^{2}}$. Using the criterion of Lawlor in [5], M. Kerckhove [4] proved the cone over an isolated orbit of the action of $S U(n)$ on the unit sphere in the vector space of traceless $n$-by- $n$ Hermitian symmetric matrices is areaminimizing for $n>2$ and the cone over an isolated orbit of the adjoint action of $S O(n)$ is area-minimizing for $n>3$.

The authors would like to thank the referee for reading carefully the manuscript and for pointing out some mistakes in it.

\section{Preliminaries}

Let $G$ be a compact connected Lie group and $\theta$ an involutive automorphism of $G$. We denote by $G_{\theta}$ the closed subgroup of all fixed points of $\theta$ in $G$. For a closed subgroup $K$ of $G$ which lies between $G_{\theta}$ and the identity component of $G_{\theta},(G, K)$ is a Riemannian symmetric pair. Let $g$ and $\mathrm{f}$ be the Lie algebras of $G$ and $K$ respectively. The involutive automorphism $\theta$ of $G$ induces an involutive automorphism of $\mathfrak{g}$, which is also denoted by $\theta$. Since $K$ lies between $G_{\theta}$ and the identity component of $G_{\theta}$, we have

$$
\mathfrak{l}=\{X \in \mathfrak{g} \mid \theta(X)=X\} .
$$

An inner product $\langle$,$\rangle on g$ which is invariant under the actions of $\operatorname{Ad}(G)$ and $\theta$ induces a bi-invariant Riemannian metric on $G$ and $G$-invariant Riemannian metric on the homogeneous space $M=G / K$, which are also denoted by the same symbol $\langle$,$\rangle . Then M$ is a compact Riemannian symmetric space with respect to $\langle$,$\rangle . Conversely any compact symmetric space is constructed in this way. Put$

$$
\mathfrak{m}=\{X \in \mathfrak{g} \mid \theta(X)=-X\} .
$$

Since $\theta$ is involutive, we have an orthogonal direct sum decomposition of $\mathfrak{g}$ :

$$
\mathfrak{g}=\mathfrak{l}+\mathbf{m} \text {. }
$$

This decomposition is called a canonical decomposition of the orthogonal symmetric Lie algebra $(\mathfrak{g}, \theta)$.

Take and fix a maximal Abelian subspace $a$ in $m$ and a maximal Abelian subalgebra $t$ in $\mathfrak{g}$ including $\mathfrak{a}$. Let $\mathfrak{c}$ be the center of $\mathfrak{g}$ and $\mathfrak{g}^{\prime}=[\mathfrak{g}, \mathfrak{g}]$. We have an 
orthogonal direct sum decomposition:

$$
\mathfrak{g}=\mathfrak{c}+\mathfrak{g}^{\prime}
$$

We set

$$
\mathfrak{a}^{\prime}=\mathfrak{a} \cap \mathfrak{g}^{\prime}, \quad \mathfrak{c}_{\mathfrak{m}}=\mathfrak{c} \cap \mathfrak{m} .
$$

We have an orthogonal direct sum decomposition:

$$
\mathfrak{a}=\mathfrak{c}_{\mathfrak{m}}+\mathfrak{a}^{\prime} .
$$

Put

$$
\mathfrak{b}=\mathfrak{t} \cap \mathfrak{l} .
$$

Since $\mathrm{t}$ is $\theta$-invariant we get an orthogonal direct sum decomposition of $\mathrm{t}$ :

$$
\mathfrak{t}=\mathfrak{b}+\mathfrak{a} .
$$

For $\alpha \in \mathrm{t}$ we put

$$
\tilde{\mathfrak{g}}_{\alpha}=\left\{X \in \mathfrak{g}^{C} \mid[H, X]=\sqrt{-1}\langle\alpha, H\rangle X(H \in \mathfrak{t})\right\}
$$

and define the root system $\tilde{R}(\mathfrak{g})$ of $\mathfrak{g}$ by

$$
\tilde{R}(\mathfrak{g})=\left\{\alpha \in \mathrm{t}-\{0\} \mid \tilde{\mathfrak{g}}_{\alpha} \neq\{0\}\right\} .
$$

We also denote $\tilde{R}$ instead of $\tilde{R}(\mathfrak{g})$. For $\alpha \in \mathfrak{a}$ we put

$$
\mathfrak{g}_{\alpha}=\left\{X \in \mathfrak{g}^{C} \mid[H, X]=\sqrt{-1}\langle\alpha, H\rangle X(H \in \mathfrak{a})\right\}
$$

and define the root system $R(\mathfrak{g}, \mathfrak{f})$ of $(\mathfrak{g}, \mathfrak{f})$ by

$$
R(\mathfrak{g}, \mathfrak{f})=\left\{\alpha \in \mathfrak{a}-\{0\} \mid \mathfrak{g}_{\alpha} \neq\{0\}\right\} .
$$

We also denote $R$ instead of $R(\mathfrak{g}, \mathfrak{f})$. Put

$$
\tilde{R}_{0}(\mathfrak{g})=\tilde{R}(\mathfrak{g}) \cap \mathfrak{b}
$$

and denote the orthogonal projection from $\mathrm{t}$ to $a$ by $H \mapsto \bar{H}$. Then we have

$$
R(\mathfrak{g}, \mathfrak{\mathfrak { l }})=\left\{\bar{\alpha} \mid \alpha \in \tilde{R}(\mathfrak{g})-\tilde{R}_{0}(\mathfrak{g})\right\} .
$$

We extend a basis of $a$ to that of $t$ and define the lexicographic orderings $>$ on $a$ and $\mathrm{t}$ with respect to these bases. Then for $H \in \mathrm{t}, \bar{H}>0$ implies $H>0$. We denote by $\tilde{F}(\mathfrak{g})$ the fundamental system of $\tilde{R}(\mathfrak{g})$ with respect to the ordering $>$. We also denote $\tilde{F}$ instead of $\tilde{F}(\mathfrak{g})$. Put

$$
\tilde{F}_{0}(\mathfrak{g})=\tilde{F}(\mathfrak{g}) \cap \tilde{R}_{0}(\mathfrak{g}) .
$$


Then the fundamental system $F(\mathfrak{g}, \mathfrak{f})$ of $R(\mathfrak{g}, \mathfrak{f})$ with respect to the ordering $>$ is given by

$$
F(\mathfrak{g}, \mathfrak{f})=\left\{\bar{\alpha} \mid \alpha \in \tilde{F}(\mathfrak{g})-\tilde{F}_{0}(\mathfrak{g})\right\} .
$$

We define positive root systems by

$$
\begin{aligned}
\tilde{R}_{+}(\mathfrak{g}) & =\{\alpha \in \tilde{R}(\mathfrak{g}) \mid \alpha>0\} \\
R_{+}(\mathfrak{g}, \mathfrak{f}) & =\{\alpha \in R(\mathfrak{g}, \mathfrak{f}) \mid \alpha>0\} .
\end{aligned}
$$

We also denote $\tilde{R}_{+}$and $R_{+}$instead of $\tilde{R}_{+}(\mathfrak{g})$ and $R_{+}(\mathfrak{g}, \mathfrak{f})$. Then

$$
R_{+}(\mathfrak{g}, \mathfrak{f})=\left\{\bar{\alpha} \mid \alpha \in \tilde{R}_{+}(\mathfrak{g})-\tilde{R}_{0}(\mathfrak{g})\right\}
$$

holds. We set

$$
\mathfrak{f}_{0}=\{X \in \mathfrak{f} \mid[X, H]=0(H \in \mathfrak{a})\}
$$

and

$$
\begin{aligned}
\mathfrak{f}_{\alpha} & =\mathfrak{f} \cap\left(\mathfrak{g}_{\alpha}+\mathfrak{g}_{-\alpha}\right) \\
\mathfrak{m}_{\alpha} & =\mathfrak{m} \cap\left(\mathfrak{g}_{\alpha}+\mathfrak{g}_{-\alpha}\right)
\end{aligned}
$$

for $\alpha \in R_{+}(\mathfrak{g}, \mathfrak{f})$. We have the following lemma ([2]).

LEMma 1.1. (1) We have orthogonal direct sum decompositions:

$$
\mathfrak{f}=\mathfrak{f}_{0}+\sum_{\alpha \in R_{+}} \mathfrak{f}_{\alpha}, \quad \mathfrak{m}=\mathfrak{a}+\sum_{\alpha \in R_{+}} \mathfrak{m}_{\alpha}
$$

(2) For each $\alpha \in \tilde{R}_{+}-\tilde{R}_{0}$ there exist $S_{\alpha} \in \mathfrak{f}$ and $T_{\alpha} \in \mathfrak{m}$ such that

$$
\left\{S_{\alpha} \mid \alpha \in \tilde{R}_{+}, \bar{\alpha}=\lambda\right\}, \quad\left\{T_{\alpha} \mid \alpha \in \tilde{R}_{+}, \bar{\alpha}=\lambda\right\}
$$

are respectively orthonormal bases of $\mathfrak{f}_{\lambda}, \mathfrak{m}_{\lambda}$ and that for $H \in \mathfrak{a}$

$$
\left[H, S_{\alpha}\right]=\langle\alpha, H\rangle T_{\alpha}, \quad\left[H, T_{\alpha}\right]=-\langle\alpha, H\rangle S_{\alpha} .
$$

We denote $m_{\lambda}=\operatorname{dim} \mathfrak{m}_{\lambda}=\operatorname{dim} \mathfrak{f}_{\lambda}$ and call it the multiplicity of $\lambda$.

We define a subset $D$ of $a$ by

$$
D=\bigcup_{\alpha \in R}\{H \in \mathfrak{a} \mid\langle\alpha, H\rangle=0\} .
$$

Each connected component of $a-D$ is called a Weyl chamber. We define the fundamental Weyl chamber by 


$$
C=\{H \in \mathfrak{a} \mid\langle\alpha, H\rangle>0(\alpha \in F(\mathfrak{g}, \mathfrak{f}))\}
$$

Its closure is given by

$$
\bar{C}=\{H \in \mathfrak{a} \mid\langle\alpha, H\rangle \geq 0(\alpha \in F(\mathfrak{g}, \mathfrak{f}))\} .
$$

For each subset $\Delta \subset F=F(\mathfrak{g}, \mathfrak{f})$ we define a subset $C^{\Delta}$ of $\bar{C}$ by

$$
C^{\Delta}=\{H \in \bar{C} \mid\langle\alpha, H\rangle>0(\alpha \in \Delta),\langle\beta, H\rangle=0(\beta \in F-\Delta)\} .
$$

We easily get the following lemma.

LeMma 1.2. (1) For $\Delta_{1} \subset F$

$$
\overline{C^{\Delta_{1}}}=\bigcup_{\Delta \subset \Delta_{1}} C^{\Delta}
$$

is a disjoint union. In particular $\bar{C}=\bigcup_{\Delta \subset F} C^{\Delta}$ is a disjoint union.

(2) For $\Delta_{1}, \Delta_{2} \subset F, \Delta_{1} \subset \Delta_{2}$ if and only if $C^{\Delta_{1}} \subset \overline{C^{\Delta_{2}}}$.

For $\beta \in F$ we take $H_{\beta} \in \mathfrak{a}^{\prime}$ satisfying the following condition.

$$
\left\langle\alpha, H_{\beta}\right\rangle= \begin{cases}1 & (\alpha=\beta) \\ 0 & (\alpha \neq \beta) .\end{cases}
$$

We have

$$
\bar{C}=\mathfrak{c}_{\mathfrak{m}} \times\left\{\sum_{\alpha \in F} t_{\alpha} H_{\alpha} \mid t_{\alpha} \geq 0\right\}
$$

and for $\Delta \subset F$

$$
C^{\Delta}=\mathfrak{c}_{\mathfrak{m}} \times\left\{\sum_{\alpha \in \Delta} t_{\alpha} H_{\alpha} \mid t_{\alpha}>0\right\} .
$$

For $H \in \mathrm{m}$ we put

$$
\begin{aligned}
& Z^{H}=\{g \in G \mid \operatorname{Ad}(g) H=H\}, \\
& Z_{K}^{H}=\{k \in K \mid \operatorname{Ad}(k) H=H\} .
\end{aligned}
$$

$Z_{K}^{H}=Z^{H} \cap K$ holds. $Z^{H}$ is a closed subgroup of $G$ and $Z_{K}^{H}$ is a closed subgroup of $K$. We can prove the following lemma by the standard argument of compact Lie groups, so we omit its proof. 
LEMMA 1.3. $Z^{H}$ is connected.

For $\Delta \subset F$ we put

$$
\begin{aligned}
& N^{\Delta}=\left\{g \in G \mid \operatorname{Ad}(g) C^{\Delta}=C^{\Delta}\right\} \\
& Z^{\Delta}=\left\{g \in G|\operatorname{Ad}(g)|_{C^{\Delta}}=1\right\} \\
& N_{K}^{\Delta}=\left\{k \in K \mid \operatorname{Ad}(k) C^{\Delta}=C^{\Delta}\right\} \\
& Z_{K}^{\Delta}=\left\{k \in K|\operatorname{Ad}(k)|_{C^{\Delta}}=1\right\} .
\end{aligned}
$$

By the above definitions we have $N_{K}^{\Delta}=N^{\Delta} \cap K$ and $Z_{K}^{\Delta}=Z^{\Delta} \cap K$. $Z^{\Delta}$ is a closed subgroup of $G$ and $Z_{K}^{\Delta}$ is a closed subgroup of $K$. If $H \in C^{\Delta}$, then

$$
Z^{\Delta} \subset Z^{H}, \quad Z_{K}^{\Delta} \subset Z_{K}^{H}
$$

We put

$$
\begin{aligned}
& R^{\Delta}=R \cap(F-\Delta)_{Z} \\
& R_{+}^{\Delta}=R^{\Delta} \cap R_{+} \\
& \mathfrak{g}^{\Delta}=\mathfrak{l}_{0}+\mathfrak{a}+\sum_{\alpha \in R_{+}^{\Delta}}\left(\mathfrak{l}_{\alpha}+\mathfrak{m}_{\alpha}\right)
\end{aligned}
$$

and

$$
\begin{gathered}
\mathfrak{l}^{\Delta}=\mathfrak{g}^{\Delta} \cap \mathfrak{f}=\mathfrak{l}_{0}+\sum_{\alpha \in R_{+}^{\Delta}} \mathfrak{l}_{\alpha} \\
\mathfrak{m}^{\Delta}=\mathfrak{g}^{\Delta} \cap \mathfrak{m}=\mathfrak{a}+\sum_{\alpha \in R_{+}^{\Delta}} \mathfrak{m}_{\alpha} .
\end{gathered}
$$

We have an orthogonal direct sum decomposition:

$$
\mathfrak{g}^{\Delta}=\mathfrak{f}^{\Delta}+\mathfrak{m}^{\Delta} \text {. }
$$

LemMa 1.4. For $\Delta \subset F$ and $H \in C^{\Delta}$, we obtain the following equations.

(1) $R_{+}^{\Delta}=\left\{\alpha \in R_{+} \mid\langle\alpha, H\rangle=0\right\}$

(2) $R^{\Delta}=\{\alpha \in R \mid\langle\alpha, H\rangle=0\}$

(3) $\mathfrak{g}^{\Delta}=\{X \in \mathfrak{g} \mid[H, X]=0\}$

Proof. Any $\alpha \in R_{+}$can be written as follows:

$$
\alpha \in \sum_{\gamma \in F} n_{\gamma} \gamma \quad\left(n_{\gamma} \in Z, n_{\gamma} \geq 0\right) .
$$


So we obtain

$$
\langle\alpha, H\rangle=\sum_{\gamma \in F} n_{\gamma}\langle\gamma, H\rangle=\sum_{\gamma \in \Delta} n_{\gamma}\langle\gamma, H\rangle
$$

From this $\langle\alpha, H\rangle=0$ if and only if $\alpha \in R_{+}^{\Delta}$. Therefore we obtain

$$
R_{+}^{\Delta}=\left\{\alpha \in R_{+} \mid\langle\alpha, H\rangle=0\right\}
$$

This implies

$$
R^{\Delta}=\{\alpha \in R \mid\langle\alpha, H\rangle=0\}
$$

Any $X \in \mathfrak{g}$ can be written as follows:

$$
X=S_{0}+\sum_{\alpha \in R_{+}} a_{\alpha} S_{\alpha}+T_{0}+\sum_{\alpha \in R_{+}} b_{\alpha} T_{\alpha}
$$

where $S_{0} \in \mathfrak{f}_{0}$ and $T_{0} \in \mathfrak{a}$. It follows from Lemma 1.1 and (1) that

$$
[H, X]=\sum_{\alpha \notin R_{+}^{\Delta}} a_{\alpha}\langle\alpha, H\rangle T_{\alpha}-\sum_{\alpha \notin R_{+}^{\Delta}} b_{\alpha}\langle\alpha, H\rangle S_{\alpha} .
$$

From this $[H, X]=0$ if and only if $X \in \mathfrak{g}^{\Delta}$. Therefore we obtain

$$
\mathfrak{g}^{\Delta}=\{X \in \mathfrak{g} \mid[H, X]=0\}
$$

Lemma 1.5. (1) Take $\Delta_{1}, \Delta_{2} \subset F, H_{1} \in C^{\Delta_{1}}, H_{2} \in C^{\Delta_{2}}$ and $g \in G$. If $\operatorname{Ad}(g) H_{1}$ $=H_{2}$, then $\operatorname{Ad}(g) \mathfrak{g}^{\Delta_{1}}=\mathfrak{g}^{\Delta_{2}}$.

(2) If $\Delta \subset F$, then $N^{\Delta} \subset N\left(\mathfrak{g}^{\Delta}\right)$. For any $H \in C^{\Delta}$, all of $Z^{H}, Z^{\Delta}, N^{\Delta}$ and $N\left(\mathfrak{g}^{\Delta}\right)$ are compact subgroups of $G$ and all of their Lie subalgebras coincide with $\mathfrak{g}^{\Delta}$.

Proof. (1) By Lemma $1.4 \mathfrak{g}^{\Delta_{1}}$ is the centralizer of $H_{1} \cdot \operatorname{Ad}(g) \mathfrak{g}^{\Delta_{1}}$ is the centralizer of $\operatorname{Ad}(g) H_{1}=H_{2}$, so is equal to $\mathrm{g}^{\Delta_{2}}$.

(2) (1) with $\Delta_{1}=\Delta_{2}=\Delta$ implies $N^{\Delta} \subset N\left(\mathfrak{g}^{\Delta}\right) . Z^{H}$ and $N\left(\mathfrak{g}^{\Delta}\right)$ are compact subgroups in $G$.

Since $\mathrm{g}^{\Delta}$ is the centralizer of $H$ by Lemma 1.4, the Lie algebra $\mathscr{L}\left(Z^{H}\right)$ of $Z^{H}$ is equal to $\mathrm{g}^{\Delta}$.

We show that the Lie algebras $\mathscr{L}\left(Z^{\Delta}\right), \mathscr{L}\left(N^{\Delta}\right)$ and $\mathscr{L}\left(N\left(\mathfrak{g}^{\Delta}\right)\right)$ of $Z^{\Delta}, N^{\Delta}$ and $N\left(\mathfrak{g}^{\Delta}\right)$ are all equal to $\mathfrak{g}^{\Delta}$. By their definitions $Z^{\Delta} \subset N^{\Delta} \subset N\left(\mathfrak{g}^{\Delta}\right) . Z^{\Delta}, N\left(\mathfrak{g}^{\Delta}\right)$ is a closed subgroup of $G$, so we have $\mathscr{L}\left(Z^{\Delta}\right) \subset \mathscr{L}\left(N\left(\mathfrak{g}^{\Delta}\right)\right)$. Any element $X$ of $\mathfrak{g}^{\Delta}$ can be written as follows: 


$$
X=S_{0}+\sum_{\alpha \in R_{+}^{\Delta}} a_{\alpha} S_{\alpha}+T_{0}+\sum_{\alpha \in R_{+}^{\Delta}} b_{\alpha} T_{\alpha} \quad\left(S_{0} \in \mathfrak{I}_{0}, T_{0} \in \mathfrak{a}\right) .
$$

From this we get $X \in \mathscr{L}\left(Z^{\Delta}\right)$, hence $\mathfrak{g}^{\Delta} \subset \mathscr{L}\left(Z^{\Delta}\right)$. Therefore

$$
\mathfrak{g}^{\Delta} \subset \mathscr{L}\left(Z^{\Delta}\right) \subset \mathscr{L}\left(N\left(\mathfrak{g}^{\Delta}\right)\right) \text {. }
$$

Conversely we assume an element

$$
X=S_{0}+\sum_{\alpha \in R_{+}} a_{\alpha} S_{\alpha}+T_{0}+\sum_{\alpha \in R_{+}} b_{\alpha} T_{\alpha} \quad\left(S_{0} \in \mathfrak{I}_{0}, T_{0} \in \mathfrak{a}\right)
$$

in $\mathfrak{g}$ is contained in $\mathscr{L}\left(N\left(\mathfrak{g}^{\Delta}\right)\right)$. Since

$$
[H, X]=\sum_{\alpha \in R_{+}} a_{\alpha}\langle\alpha, H\rangle T_{\alpha}-\sum_{\alpha \in R_{+}} b_{\alpha}\langle\alpha, H\rangle S_{\alpha}
$$

we obtain $X \in \mathrm{g}^{\Delta}$. Therefore we get

$$
\mathfrak{g}^{\Delta}=\mathscr{L}\left(Z^{\Delta}\right)=\mathscr{L}\left(N\left(\mathfrak{g}^{\Delta}\right)\right) .
$$

$N^{\Delta}$ satisfies $Z^{\Delta} \subset N^{\Delta} \subset N\left(\mathfrak{g}^{\Delta}\right)$ and $\mathscr{L}\left(Z^{\Delta}\right)=\mathscr{L}\left(N\left(\mathfrak{g}^{\Delta}\right)\right)$. So $N^{\Delta}$ is also a compact subgroup of $G$ and $\mathscr{L}\left(N^{\Delta}\right)=\mathfrak{g}^{\Delta}$ holds.

THEOREM 1.6. For any $\Delta \subset F$ and $H \in C^{\Delta}$

$$
Z^{\Delta}=Z^{H}=N^{\Delta}, \quad Z_{K}^{\Delta}=Z_{K}^{H}=N_{K}^{\Delta} .
$$

Proof. By the definition we have $Z^{\Delta} \subset Z^{H}$ and by the above lemma their Lie algebras coincides. Moreover $Z^{H}$ is connected by Lemma 1.3, so we obtain $Z^{\Delta}=Z^{H}$.

$Z^{\Delta}$ and $N^{\Delta}$ are compact and have the same Lie algebra $\mathrm{g}^{\Delta}$. Since $Z^{\Delta}$ is the kernel of the homomorphism

$$
\text { Ad }: N^{\Delta} \rightarrow \text { The permutation group of } C^{\Delta},
$$

$Z^{\Delta}$ is a normal subgroup of $N^{\Delta}$ and $N^{\Delta} / Z^{\Delta}$ is a finite group. For any $g \in N^{\Delta}$, the action of $\operatorname{Ad}(g)$ on $C^{\Delta}$ has a finite order, that is, there is an integer $N$ satisfying $\left.\operatorname{Ad}(g)^{N}\right|_{C^{\Delta}}=1$. Take $H_{0} \in C^{\Delta}$ and put

$$
H_{1}=\frac{1}{N}\left(H_{0}+\operatorname{Ad}(g) H_{0}+\cdots+\operatorname{Ad}(g)^{N-1} H_{0}\right) .
$$

Each $\operatorname{Ad}(g)^{i} H_{0}$ is contained in $C^{\Delta}$ and $C^{\Delta}$ is convex, so we get $H_{1} \in C^{\Delta}$. $\operatorname{Ad}(g) H_{1}=H_{1}$ holds and $g \in Z^{H_{1}}=Z^{\Delta}$. Hence $N^{\Delta} \subset Z^{\Delta}$ and we obtain $Z^{\Delta}=N^{\Delta}$.

The second equation follows from the first one. 


\section{Construction of Retractions}

The notation of the preceding section will be preserved. Let $B$ be a compact submanifold of the unit sphere in $\boldsymbol{R}^{n}$. We call $C_{B}=\{t x \mid x \in B, t \geq 0\}$ the cone over $B$. $C_{B}$ is said to be area-minimizing if $C_{B}^{1}=\{t x \mid x \in B, 0 \leq t \leq 1\}$ has the least area among all surfaces with boundary $B$.

For an unit vector $H \in \bar{C}$, the orbit $\operatorname{Ad}(K) H$ is a submanifold of the unit sphere in $m$. Then the mapping

$$
f: k Z_{K}^{H} \mapsto \operatorname{Ad}(k) H
$$

is a diffeomorphism of the homogeneous space $K / Z_{K}^{H}$ to $\operatorname{Ad}(K) H$.

Proposition 2.1. The orbit $\operatorname{Ad}(K) H$ is connected.

ProOF. Since $\mathrm{m}=\bigcup_{k \in K_{0}} \operatorname{Ad}(k) \cdot \mathfrak{a}$, where $K_{0}$ be the identity component of $K$, for any $\operatorname{Ad}(k) H \in \operatorname{Ad}(K) H$ there exists an element $k_{1} \in K_{0}$ such that $\operatorname{Ad}\left(k_{1} k\right) H \in \mathfrak{a}$. From Proposition 2.2 (p. 285) of [2], there exists a member of Weyl group whose action on $\mathfrak{a}$ is represented by $\operatorname{Ad}\left(k_{2}\right)$ for some $k_{2} \in K_{0}$ such that $\operatorname{Ad}\left(k_{1} k\right) H=$ $\operatorname{Ad}\left(k_{2}\right) H$. If we put $k_{0}=k_{1}^{-1} k_{2} \in K_{0}$, then $\operatorname{Ad}(k) H=\operatorname{Ad}\left(k_{0}\right) H$ holds. Thus we get $\operatorname{Ad}(K) H=\operatorname{Ad}\left(K_{0}\right) H$ and it is connected.

From now on we assume that $(G, K)$ is irreducible.

Let $F(\mathfrak{g}, \mathfrak{f})=\left\{\alpha_{1}, \ldots, \alpha_{l}\right\}$ be the fundamental root system and $\tilde{\alpha}=$ $n_{1} \alpha_{1}+\cdots+n_{l} \alpha_{l}$ be the highest root of $R(\mathfrak{g}, \mathfrak{f})$. Select $\alpha_{i} \in F(\mathfrak{g}, \mathfrak{f})$ such that $n_{i}=1$, we put $\alpha_{0}=\alpha_{i}$ and $A_{0}=H_{\alpha_{0}} /\left|H_{\alpha_{0}}\right|$. It is known that $f$ is an isometry of $K / Z_{K}^{A_{0}}$ with the normal homogeneous Riemannian metric multiplied some constant onto $\operatorname{Ad}(K) A_{0}$ and that $K / Z_{K}^{A_{0}}$ is a symmetric space. We call this space a symmetric $R$-space, and $f$ its canonical imbedding. Because $\operatorname{Ad}(K) A_{0}$ is an isolated orbit, $\operatorname{Ad}(K) A_{0}$ is a minimal submanifold of the unit sphere in $\mathrm{m}$ by a result of Hsiang [3]. Hence the cone $C_{\operatorname{Ad}(K) A_{0}}$ is also a minimal submanifold of $\mathrm{m}$ ([6] p. 97, Prop. 6.1.1). The purpose of this article is to prove $C_{\operatorname{Ad}(K) A_{0}}$ is an area-minimizing cone.

Proposition 2.2. Let $V$ and $W$ be two vector spaces with inner products. Suppose $n=\operatorname{dim} W \leq \operatorname{dim} V$. For a linear mapping $F$ of $V$ to $W$ we put

$$
J F=\sup \left\{\left|F\left(u_{1}\right) \wedge \cdots \wedge F\left(u_{n}\right)\right|\right\}
$$

where $u_{1}, \ldots, u_{n}$ runs over all orthonormal vectors of $V$. If $F$ is not surjective, then 
$J F=0$. If $F$ is surjective, then $J F$ coincides with

$$
\left|F\left(v_{1}\right) \wedge \cdots \wedge F\left(v_{n}\right)\right|
$$

for an orthonormal base $v_{1}, \ldots, v_{n}$ of $(\operatorname{ker} F)^{\perp}$.

Let $B$ be a compact submanifold of the unit sphere in $m$. We call a differentiable retraction $\Phi: m \rightarrow C_{B}$ a area-nonincreasing if

$$
J\left(d \Phi_{x}\right) \leq 1
$$

for $x \in \mathbf{m}$.

Proposition 2.3. The cone $C_{B}$ over a compact submanifold $B$ of the unit sphere in $m$ is area-minimizing if there exists an area-nonincreasing retraction $\Phi: \mathfrak{m} \rightarrow C_{B}$.

Proof. Let $S$ be a surface in $m$ with boundary $B$. Since $C_{B}^{1} \subset \Phi(S)$, we have $\operatorname{vol}\left(C_{B}^{1}\right) \leq \operatorname{vol}(\Phi(S))$. Let $e_{1}, \ldots, e_{n}$ be an orthonormal frame of $S$, then

$$
\begin{aligned}
\operatorname{vol}(\Phi(S)) & =\int_{S}\left|d \Phi\left(e_{1} \wedge \cdots \wedge e_{n}\right)\right| d \mu_{S} \\
& \leq \int_{S} J\left(d \Phi_{x}\right) d \mu_{S} \\
& \leq \int_{S} d \mu_{S} \\
& =\operatorname{vol}(S) .
\end{aligned}
$$

Consequently,

$$
\operatorname{vol}\left(C_{B}^{1}\right) \leq \operatorname{vol}(\Phi(S)) \leq \operatorname{vol}(S)
$$

This proves the proposition.

We shall now consider a way to construct area-nonincreasing retractions.

LEMMA 2.4. Suppose $\phi$ is a mapping of $\bar{C}$ into itself such that $\phi\left(C^{\Delta}\right) \subset \overline{C^{\Delta}}$ for each $\Delta \subset F(\mathfrak{g}, \mathfrak{f})$. Then $\phi$ extends to a mapping $\Phi$ of $\mathrm{m}$ as

$$
\Phi(X)=\operatorname{Ad}(k) \phi(H),
$$

for each $X=\operatorname{Ad}(k) H \in \mathfrak{m}(k \in K, H \in \bar{C})$. 
Proof. Suppose $k_{1}, k_{2} \in K$ and $H_{1}, H_{2} \in \bar{C}$ satisfy $\operatorname{Ad}\left(k_{1}\right) H_{1}=\operatorname{Ad}\left(k_{2}\right) H_{2}$. Then $\operatorname{Ad}\left(k_{2}^{-1} k_{1}\right) H_{1}=H_{2} \in \mathfrak{a}$ and we have $H_{1}=H_{2}$, because each orbit of the Weyl group on $a$ intersects $\bar{C}$ in exactly one point ([3], p. 293, Th. 2.22). Let

$$
\Delta=\left\{\alpha \in F \mid\left\langle\alpha, H_{1}\right\rangle>0\right\} .
$$

We have $H_{1} \in C^{\Delta}$ and $\phi\left(H_{1}\right) \in \overline{C^{\Delta}}$ by the assumption of $\phi$. Thus Theorem 1.6 implies

$$
k_{2}^{-1} k_{1} \in Z_{K}^{H_{1}}=Z_{K}^{\Delta}
$$

therefore $\operatorname{Ad}\left(k_{2}^{-1} k_{1}\right) \phi\left(H_{1}\right)=\phi\left(H_{1}\right)$.

From Lemma 2.4, we have the following.

Proposition 2.5. Let $\phi: \bar{C} \rightarrow\left\{t A_{0} \mid t \geq 0\right\}$ be a differentiable mapping. Denote $\phi(x)=f(x) A_{0}$. If $f$ satisfies $f\left(t A_{0}\right)=t(t \geq 0)$ and $\left.f\right|_{\left\{\alpha_{0}\right\}^{\perp}} \equiv 0$, then $\phi$ extends to a differentiable retraction $\Phi: \mathrm{m} \rightarrow C_{\mathrm{Ad}(K) A_{0}}$.

In this case $\Phi$ is area-nonincreasing if and only if (1) holds for each $x \in C$.

We will compute $J\left(d \Phi_{x}\right)$ of $\Phi$ in Proposition 2.5 for $x \in C$.

Proposition 2.6. We denote $R_{+}\left(A_{0}\right)=\left\{\lambda \in R_{+} \mid\left\langle\lambda, A_{0}\right\rangle>0\right\}$.

$$
J\left(d \Phi_{x}\right)=|\operatorname{grad}(f)| \prod_{\lambda \in R_{+}\left(A_{0}\right)}\left(\frac{\left\langle\lambda, A_{0}\right\rangle}{\langle\lambda, x\rangle} f(x)\right)^{m_{\lambda}} .
$$

Proof. If $f(x)=0$, then the both sides of the equation are 0 . So we consider the case $f(x) \neq 0$. By the definition of $\Phi, d \Phi_{x}(\mathfrak{a}) \subset \boldsymbol{R} A_{0}$. By using the equation

$$
\left.\frac{d}{d t}\right|_{t=0} \operatorname{Ad}\left(\exp t S_{\alpha}\right) x=-\langle\alpha, x\rangle T_{\alpha}
$$

for $\alpha \in \tilde{R}_{+}(\mathfrak{g}), \bar{\alpha} \neq 0$, we have

$$
d \Phi_{x}\left(T_{\alpha}\right)=\frac{\langle\alpha, \phi(x)\rangle}{\langle\alpha, x\rangle} T_{\alpha}
$$

From this we get

$$
d \Phi_{x}\left(\sum_{\lambda \in R_{+}} \mathfrak{m}_{\lambda}\right) \subset \sum_{\lambda \in R_{+}\left(A_{0}\right)} \mathfrak{m}_{\lambda}
$$


so we can write

$$
\begin{aligned}
J\left(d \Phi_{x}\right) & =J_{1}(x) \cdot J_{2}(x), \\
J_{1}(x) & =J\left(d \Phi_{x} \mid \mathfrak{a}\right), \\
J_{2}(x) & =J\left(d \Phi_{x} \mid \sum \mathfrak{m}_{\lambda}\right) .
\end{aligned}
$$

Take a unit vector $v$ in $\mathfrak{a}$.

$$
\begin{aligned}
d \Phi_{x}(v) & =d \phi_{x}(v) \\
& =d f_{x}(v) A_{0} \\
& =\langle\operatorname{grad}(f), v\rangle A_{0} .
\end{aligned}
$$

Therefore

$$
\begin{aligned}
J_{1}(x) & =\max \left\{\left|d \Phi_{x}(v)\right||v \in \mathfrak{a},| v \mid=1\right\} \\
& =|\operatorname{grad}(f)| .
\end{aligned}
$$

Secondly for $J_{2}(x)$, we see the kernel of $\left.d \Phi_{x}\right|_{\sum m_{\lambda}}$. By the above expression of $d \Phi_{x}\left(T_{\alpha}\right)$ we get

$$
\operatorname{ker}\left(d \Phi_{x} \mid \sum \mathfrak{m}_{\lambda}\right)=\sum_{\substack{\lambda \in R_{+} \\\left\langle\lambda, A_{0}\right\rangle=0}} \mathfrak{m}_{\lambda}
$$

We can take $\left\{T_{\alpha} \mid \alpha \in \tilde{R}_{+}(\mathfrak{g}),\left\langle\alpha, A_{0}\right\rangle>0\right\}$ as an orthonormal base of $\operatorname{ker}\left(\left.d \Phi_{x}\right|_{\sum m_{\lambda}}\right)^{\perp}$. It follows that

$$
J_{2}(x)=\left|\bigwedge_{\substack{\alpha \in \tilde{R}_{+} \\\left\langle\alpha, A_{0}\right\rangle>0}} d \Phi_{x}\left(T_{\alpha}\right)\right|=\prod_{\lambda \in R_{+}\left(A_{0}\right)}\left(\frac{\left\langle\lambda, A_{0}\right\rangle}{\langle\lambda, x\rangle} f(x)\right)^{m_{\lambda}}
$$

where $m_{\lambda}$ is the multiplicity of $\lambda$. So we have

$$
J\left(d \Phi_{x}\right)=|\operatorname{grad}(f)| \prod_{\lambda \in R_{+}\left(A_{0}\right)}\left(\frac{\left\langle\lambda, A_{0}\right\rangle}{\langle\lambda, x\rangle} f(x)\right)^{m_{\lambda}} .
$$

\section{Construction of Area-nonincreasing Retractions}

THEOREM 3.1. The cones over

$$
\frac{S O(2 l+1)}{S O(2) \times S O(2 l-1)}, \quad \frac{S O(l) \times S O(l+n)}{S^{\prime}(O(l-1) \times O(l+n-1))}
$$


corresponding to symmetric pairs

$$
\left(S O(2 l+1)^{2}, S O(2 l+1)\right), \quad(S O(2 l+n), S O(l) \times S O(l+n)) \quad(n \geq 2)
$$

respectively are area-minimizing, where

$$
\begin{aligned}
& S^{\prime}(O(l-1) \times O(l+n-1))
\end{aligned}
$$

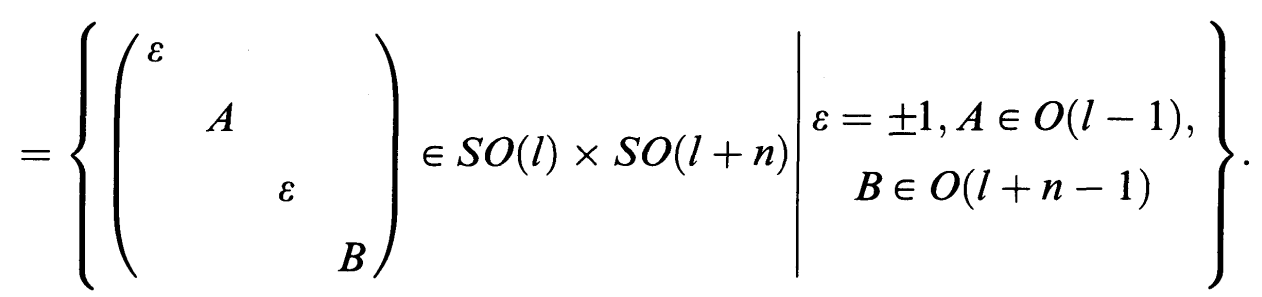

Proof. We consider symmetric pairs of type $B_{l}$. Let $\varepsilon_{1}, \ldots, \varepsilon_{l}$ be an orthonormal basis of the maximal Abelian subspace $\mathfrak{a}$ such that all roots are

$$
\pm \varepsilon_{i} \pm \varepsilon_{j}(1 \leq i<j \leq l), \quad \pm \varepsilon_{i}(1 \leq i \leq l) \text {. }
$$

Then for a suitable ordering

$$
\begin{aligned}
F(\mathfrak{g}, \mathfrak{f}) & =\left\{\alpha_{1}, \alpha_{2}, \ldots, \alpha_{l-1}, \alpha_{l}\right\}, \\
\alpha_{i} & =\varepsilon_{i}-\varepsilon_{i+1} \quad(1 \leq i<l), \quad \alpha_{l}=\varepsilon_{l}, \\
H_{\alpha_{i}} & =\varepsilon_{1}+\cdots+\varepsilon_{i}(1 \leq i \leq l), \\
\tilde{\alpha} & =\alpha_{1}+2 \alpha_{2}+\cdots+2 \alpha_{l}=\varepsilon_{1}+\varepsilon_{2}
\end{aligned}
$$

and we put

$$
A_{0}=\frac{H_{\alpha_{1}}}{\left|H_{\alpha_{1}}\right|}=\varepsilon_{1} .
$$

We have

$$
\begin{aligned}
R_{+}\left(A_{0}\right)= & \left\{\sum_{i=1}^{l} \alpha_{i}\right\} \cup\left\{\sum_{i=1}^{k-1} \alpha_{i} \mid 2 \leq k \leq l\right\} \\
& \cup\left\{\tilde{\alpha}-\sum_{i=2}^{k} \alpha_{i} \mid 2 \leq k \leq l-1\right\} \cup\{\tilde{\alpha}\}
\end{aligned}
$$

Because the multiplicities of roots of same length coincide with each other, we can denote by $m_{1}$ the multiplicity of the $\sum_{i=1}^{l} \alpha_{i}$ and by $m_{2}$ the multiplicity of the rest. For $x=\sum_{i=1}^{l} x_{i} H_{\alpha_{i}} \in \bar{C}$ we define 


$$
f(x)=\sqrt{\left\langle\alpha_{1}, x\right\rangle\langle\tilde{\alpha}, x\rangle}=\sqrt{x_{1}\left(x_{1}+2 x_{2}+\cdots+2 x_{l}\right)}
$$

then it satisfies Proposition 2.5. Using (2), we calculate $J\left(d \Phi_{x}\right)$. Since

$$
\begin{aligned}
& \frac{\partial f}{\partial x_{1}}=\frac{x_{1}+\cdots+x_{l}}{f}, \\
& \frac{\partial f}{\partial x_{i}}=\frac{x_{1}}{f} \quad(2 \leq i \leq l),
\end{aligned}
$$

we get

$$
J_{1}(x)=|\operatorname{grad}(f)|=\left|\sum_{i=1}^{l} \frac{\partial f}{\partial x_{i}} \alpha_{i}\right|=\sqrt{\frac{\left\langle\alpha_{1}, x\right\rangle^{2}+\langle\tilde{\alpha}, x\rangle^{2}}{2\left\langle\alpha_{1}, x\right\rangle\langle\tilde{\alpha}, x\rangle}} .
$$

We also obtain

$$
J_{2}(x)=\left(\frac{f}{\left\langle\sum_{i=1}^{l} \alpha_{i}, x\right\rangle}\right)^{m_{1}} \times \prod_{k=2}^{l-1}\left(\frac{f^{2}}{\left\langle\sum_{i=1}^{k} \alpha_{i}, x\right\rangle\left\langle\tilde{\alpha}-\sum_{i=2}^{k} \alpha_{i}, x\right\rangle}\right)^{m_{2}} .
$$

Therefore

$$
\begin{aligned}
J\left(d \Phi_{x}\right)= & \sqrt{\frac{\left\langle\alpha_{1}, x\right\rangle^{2}+\langle\tilde{\alpha}, x\rangle^{2}}{2\left\langle\alpha_{1}, x\right\rangle\langle\tilde{\alpha}, x\rangle}\left(\frac{\sqrt{\left\langle\alpha_{1}, x\right\rangle\langle\tilde{\alpha}, x\rangle}}{\left\langle\sum_{i=1}^{l} \alpha_{i}, x\right\rangle}\right)^{m_{1}}} \\
& \times \prod_{k=2}^{l-1}\left(\frac{\left\langle\alpha_{1}, x\right\rangle\langle\tilde{\alpha}, x\rangle}{\left\langle\sum_{i=1}^{k} \alpha_{i}, x\right\rangle\left\langle\tilde{\alpha}-\sum_{i=2}^{k} \alpha_{i}, x\right\rangle}\right)^{m_{2}} .
\end{aligned}
$$

For any $k$,

$$
\begin{aligned}
& \left\langle\sum_{i=1}^{k} \alpha_{i}, x\right\rangle\left\langle\tilde{\alpha}-\sum_{i=2}^{k} \alpha_{i}, x\right\rangle \\
& =\left\langle\sum_{i=1}^{k} \alpha_{i}, x\right\rangle\langle\tilde{\alpha}, x\rangle-\left\langle\sum_{i=1}^{k} \alpha_{i}, x\right\rangle\left\langle\sum_{i=2}^{k} \alpha_{i}, x\right\rangle \\
& =\left\langle\alpha_{1}, x\right\rangle\langle\tilde{\alpha}, x\rangle+\left\langle\sum_{i=2}^{k} \alpha_{i}, x\right\rangle\langle\tilde{\alpha}, x\rangle-\left\langle\sum_{i=1}^{k} \alpha_{i}, x\right\rangle\left\langle\sum_{i=2}^{k} \alpha_{i}, x\right\rangle \\
& =\left\langle\alpha_{1}, x\right\rangle\langle\tilde{\alpha}, x\rangle+\left\langle\sum_{i=2}^{k} \alpha_{i}, x\right\rangle\left(\langle\tilde{\alpha}, x\rangle-\left\langle\sum_{i=1}^{k} \alpha_{i}, x\right\rangle\right) \\
& \geq\left\langle\alpha_{1}, x\right\rangle\langle\tilde{\alpha}, x\rangle,
\end{aligned}
$$


hence

$$
\frac{\left\langle\alpha_{1}, x\right\rangle\langle\tilde{\alpha}, x\rangle}{\left\langle\sum_{i=1}^{k} \alpha_{i}, x\right\rangle\left\langle\tilde{\alpha}-\sum_{i=2}^{k} \alpha_{i}, x\right\rangle} \leq 1 .
$$

On the other hand

$$
\begin{aligned}
& \left\langle\sum_{i=1}^{l} \alpha_{i}, x\right\rangle^{2}-\left\langle\alpha_{1}, x\right\rangle\langle\tilde{\alpha}, x\rangle \\
& =\left\langle\sum_{i=1}^{l} \alpha_{i}, x\right\rangle^{2}-\left\langle\alpha_{1}, x\right\rangle\left\langle\sum_{i=1}^{l} \alpha_{i}+\sum_{i=2}^{l} \alpha_{i}, x\right\rangle \\
& =\left\langle\sum_{i=1}^{l} \alpha_{i}, x\right\rangle\left\langle\sum_{i=2}^{l} \alpha_{i}, x\right\rangle-\left\langle\alpha_{1}, x\right\rangle\left\langle\sum_{i=2}^{l} \alpha_{i}, x\right\rangle \\
& =\left\langle\sum_{i=2}^{l} \alpha_{i}, x\right\rangle^{2} \geq 0 .
\end{aligned}
$$

Therefore

$$
\frac{\sqrt{\left\langle\alpha_{1}, x\right\rangle\langle\tilde{\alpha}, x\rangle}}{\left\langle\sum_{i=1}^{l} \alpha_{i}, x\right\rangle} \leq 1 .
$$

We consider the case $m_{1} \geq 2$ and let

$$
A=J_{1}(x)\left(\frac{\sqrt{\left\langle\alpha_{1}, x\right\rangle\langle\tilde{\alpha}, x\rangle}}{\left\langle\sum_{i=1}^{l} \alpha_{i}, x\right\rangle}\right)^{2} .
$$

If $A \leq 1$ then $J\left(d \Phi_{x}\right) \leq 1$.

$$
\begin{aligned}
A^{2} & =\frac{\left\langle\alpha_{1}, x\right\rangle^{2}+\langle\tilde{\alpha}, x\rangle^{2}}{2\left\langle\alpha_{1}, x\right\rangle\langle\tilde{\alpha}, x\rangle} \frac{\left\langle\alpha_{1}, x\right\rangle^{2}\langle\tilde{\alpha}, x\rangle^{2}}{\left\langle\sum_{i=1}^{l} \alpha_{i}, x\right\rangle^{4}} \\
& =\frac{8\left(\left\langle\alpha_{1}, x\right\rangle^{3}\langle\tilde{\alpha}, x\rangle+\left\langle\alpha_{1}, x\right\rangle\langle\tilde{\alpha}, x\rangle^{3}\right)}{\left(\langle\tilde{\alpha}, x\rangle+\left\langle\alpha_{1}, x\right\rangle\right)^{4}} .
\end{aligned}
$$

Here subtracting the numerator from the denominator we have

$$
\begin{gathered}
\left(\langle\tilde{\alpha}, x\rangle+\left\langle\alpha_{1}, x\right\rangle\right)^{4}-8\left(\left\langle\alpha_{1}, x\right\rangle^{3}\langle\tilde{\alpha}, x\rangle+\left\langle\alpha_{1}, x\right\rangle\langle\tilde{\alpha}, x\rangle^{3}\right) \\
=\left(\langle\tilde{\alpha}, x\rangle-\left\langle\alpha_{1}, x\right\rangle\right)^{4} \geq 0 .
\end{gathered}
$$

Therefore if $m_{1} \geq 2$, we get $J\left(d \Phi_{x}\right) \leq 1$. 
There are two kind of symmetric pairs: (i) $m_{1}=n(n \geq 2), m_{2}=1$ and (ii) $m_{1}=m_{2}=2$.

(i) $(G, K)=(S O(2 l+n), S O(l) \times S O(l+n))$. It is defined by the involution:

$$
\theta(g)=I_{l, l+n} g I_{l, l+n} \quad(g \in S O(2 l+n)), \quad I_{l, l+n}=\left(\begin{array}{cc}
-I_{l} & 0 \\
0 & I_{l+n}
\end{array}\right)
$$

Then

$$
\begin{aligned}
& \mathfrak{g}=\mathfrak{s o}(2 l+n)=\left\{X \in M_{2 l+n}(\boldsymbol{R}) \mid X+{ }^{t} X=0\right\} \\
& \mathfrak{f}=\mathfrak{s o}(l) \times \mathfrak{s o}(l+n)=\left\{\left(\begin{array}{ll}
x & 0 \\
0 & y
\end{array}\right) \mid x \in \mathfrak{s} \mathfrak{o}(l), y \in \mathfrak{s o}(l+n)\right\} \\
& \mathfrak{m}=\left\{\left(\begin{array}{cc}
0 & x \\
-{ }^{t} x & 0
\end{array}\right) \mid x \in M_{l, l+n}(\boldsymbol{R})\right\} \cong M_{l, l+n}(\boldsymbol{R}) .
\end{aligned}
$$

Since $\mathrm{m}$ is isomorphic to $M_{l, l+n}(\boldsymbol{R})$, we identify them. The action of $K$ on $\mathrm{m}$ through this identification is

$$
\operatorname{Ad}\left(\begin{array}{cc}
k & 0 \\
0 & k^{\prime}
\end{array}\right) \cdot x=k x^{t} k^{\prime}
$$

We define an $\operatorname{Ad}(K)$-invariant inner product on $\mathfrak{m}$ by

$$
\langle X, Y\rangle=\operatorname{Trace}\left({ }^{t} X Y\right) \quad(X, Y \in \mathbf{m}) .
$$

Let

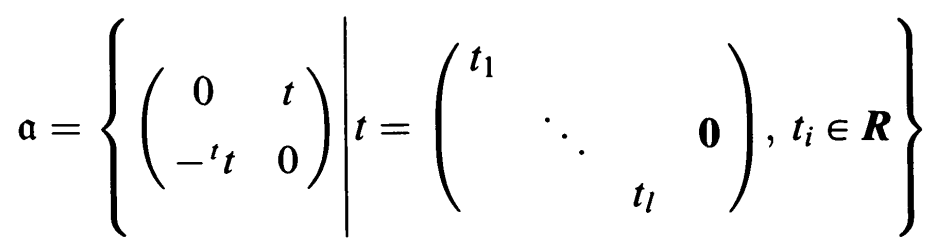

$$
\begin{aligned}
& \cong\left\{t=\left(\begin{array}{llll}
t_{1} & & & \\
& \ddots & & \mathbf{0} \\
& & t_{l}
\end{array}\right)\right\} .
\end{aligned}
$$

Then $\mathfrak{a}$ is a maximal Abelian subspace in $m$. Next, we consider a root space decomposition of $\mathrm{m}$ with respect to a. Let $E_{p q}$ be a matrix whose $(p, q)$-entry is 1 and all other entries are 0 . Then 


$$
\begin{aligned}
\varepsilon_{p} & =E_{p p}, \\
\mathfrak{m}_{\varepsilon_{p}} & =\sum_{q=l+1}^{l+n} \boldsymbol{R} E_{p q}, \\
\mathfrak{m}_{\varepsilon_{p}-\varepsilon_{q}} & =\boldsymbol{R}\left(E_{p q}+E_{q p}\right), \\
\mathfrak{m}_{\varepsilon_{p}+\varepsilon_{q}} & =\boldsymbol{R}\left(E_{p q}-E_{q p}\right) .
\end{aligned}
$$

Since $A_{0}=\varepsilon_{1}=E_{11}$, we have

$$
Z_{K}^{A_{0}}=S^{\prime}(O(l-1) \times O(l+n-1)) .
$$

Hence the corresponding symmetric $R$-space is

$$
\frac{S O(l) \times S O(l+n)}{S^{\prime}(O(l-1) \times O(l+n-1))} \text {. }
$$

(ii) $(G, K)=\left(S O(2 l+1)^{2}, S O(2 l+1)\right)$. It is defined by the involution such that

$$
\theta\left(g_{1}, g_{2}\right)=\left(g_{2}, g_{1}\right) \quad\left(\left(g_{1}, g_{2}\right) \in S O(2 l+1)\right)
$$

Then

$$
\begin{aligned}
\mathfrak{g} & =\mathfrak{s} \mathfrak{D}(2 l+1) \times \mathfrak{s o}(2 l+1) \\
\mathfrak{l} & =\{(x, x) \mid x \in \mathfrak{s o}(2 l+1)\}, \\
\mathfrak{m} & =\{(x,-x) \mid x \in \mathfrak{s o}(2 l+1)\} \cong \mathfrak{s o}(2 l+1) .
\end{aligned}
$$

Since $\mathfrak{m}$ is isomorphic to $\mathfrak{s o}(2 l+1)$, we identify them. The action of $K$ on $\mathfrak{m}$ through this identification is

$$
\operatorname{Ad}(k) \cdot x=k x^{t} k \quad(k \in S O(2 l+1), x \in \mathfrak{s} \mathfrak{D}(2 l+1)) .
$$

We define an $\operatorname{Ad}(K)$-invariant inner product on $m$ by

$$
\langle X, Y\rangle=\frac{1}{2} \operatorname{Trace}\left({ }^{t} X Y\right) \quad(X, Y \in \mathrm{m}) .
$$

Let

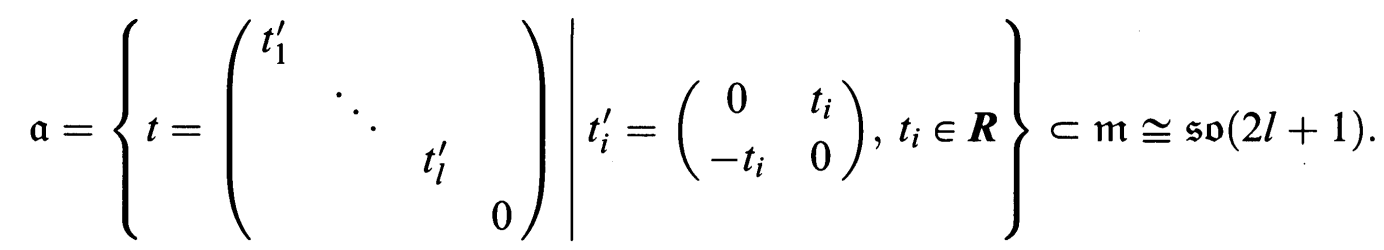


Then $\mathfrak{a}$ is a maximal Abelian subspace in $m$. Next, we consider a root space decomposition of $m$ with respect to a. Let $G_{p q}=E_{p q}-E_{q p}$. Then

$$
\begin{aligned}
\varepsilon_{p} & =G_{2 p-1,2 p}, \\
\mathfrak{m}_{\varepsilon_{p}} & =\boldsymbol{R} G_{2 p-1,2 l+1}+\boldsymbol{R} G_{2 p, 2 l+1}, \\
\mathfrak{m}_{\varepsilon_{p}-\varepsilon_{q}} & =\boldsymbol{R}\left(G_{2 p-1,2 q-1}-G_{2 p, 2 q}\right)+\boldsymbol{R}\left(G_{2 p-1,2 q}+G_{2 p, 2 q-1}\right), \\
\mathfrak{m}_{\varepsilon_{p}+\varepsilon_{q}} & =\boldsymbol{R}\left(G_{2 p-1,2 q-1}+G_{2 p, 2 q}\right)+\boldsymbol{R}\left(G_{2 p-1,2 q}-G_{2 p, 2 q-1}\right) .
\end{aligned}
$$

Since $A_{0}=\varepsilon_{1}=G_{12}$, we have

$$
Z_{K}^{A_{0}}=S O(2) \times S O(2 l-1) .
$$

Hence the corresponding symmetric $R$-space is

$$
\frac{S O(2 l+1)}{S O(2) \times S O(2 l-1)}
$$

\section{References}

[ 1 ] B. N. Cheng, Area-minimizing cone-type surfaces and coflat calibrations, Indiana Math. Univ. J. 37 (1988), 505-535.

[2] S. Helgason, Differential geometry, Lie groups, and symmetric spaces, Academic Press, (1978).

[ 3 ] W. Y. Hsiang, On compact homogeneous submanifolds. Proc. Nat. Acad. Sci. U.S.A. 56 (1966), $5-6$.

[4] M. Kerckhove, Isolated orbits of the adjoint action and area-minimizing cones, Proc. Amer. Math. Soc. 121 (1994), 497-503.

[ 5] G. R. Lawlor, A sufficient criterion for a cone to be area-minimizing, Mem. Amer. Math. Soc. 91 no. 446, 1991.

[6] J. Simons, Minimal varieties in riemannian manifolds, Ann. of Math. 88 (1968), 62-105.

Institute of Mathematics

University of Tsukuba

Tsukuba, Ibaraki

305-8571

Japan

daigo@math.tsukuba.ac.jp

kanno@math.tsukuba.ac.jp tasaki@math.tsukuba.ac.jp 